$92.85 \%$. The diagnostic accuracy of the staining was $85.93 \%$ for galectin-3, 86.15\% for HBME-1 and $87.50 \%$ for both markers. Conclusion: Galectin-3 and HBME-1 have an excellent sensitivity and specificity for malignant thyroid lesions. Combining these markers could increase sensitivity and could be useful as an adjunct to distinguish benign from malignant thyroid lesion.

\section{THE ROLE OF PCR ON DIAGNOSIS OF SMALL B- CELL NON-HODGKIN LYMPHOMA}

Ery Kus Dwianingsih, Ester Lianawati Antoro, Indrawati, F.X. Ediati Triningsih, Harijadi

Department of Anatomical Pathology, Faculty of Medicine, Universitas Gadjah Mada, Yogyakarta, Indonesia

Small B-cell non-Hodgkin lymphoma (NHL) is difficult to be distinguished from non-neoplastic reactive process using conventional haematoxylin and eosin (H\&E) staining due to different interpretation among pathologists to diagnose based on morphologic features. Ancillary examination such as immunohistochemical (IHC) staining is essential to be conducted. However, a negative or doubtful result is sometimes obtained due to unsatisfying tissue processing or IHC technique. Polymerase chain reaction (PCR) as a molecular diagnostic technique is very sensitive and specific. Clonality detection of heavy chain immunoglobulin $(\operatorname{IgH})$ gene rearrangement has been widely used to establish diagnosis of B-cell NHL.

Aims: To elaborate interobserver variation in small B-cell NHL diagnosis based on morphologic features only and to confirm sensitivity and specificity of PCR technique as an ancillary method.

Methods: The 28 samples of small B cell NHL and suspicious lymphoma were interpreted by 3 pathologists in Sardjito General Hospital based on their morphology only. The reliability of assessment and the coefficient of interobserver agreement were calculated by Fleiss kappa statistic. Interpretation results were confirmed with IHC staining (CD20, CD3, $\mathrm{Bcl} 2$ ). PCR was performed to analyze the clonality of $\operatorname{IgH}$ gene rearrangement.

Results: Interobserver agreement in morphologic evalution of small B cell NHL and chronic lymphadenitis revealed kappa coefficient 0.69 included in substantial agreement category. The cases were divided into 3 groups based on morphology and IHC result; lymphoma, reactive process; and undetermined group. PCR analysis showed $90 \%$ sensitivity and $60 \%$ specificity.

Conclusions: The present study reveals a substantial agreement among pathologists on small B-cell NHL diagnosis. On difficult cases, PCR is useful as a complementary method for morphologic and IHC examinations to establish definitive diagnosis.

\section{EVALUATION OF THE ROLE OF IMMUNOHISTOCHEMICAL EXPRESSION OF EGFR (ERB B1) AND HER4 (ERB B4) IN URINARY BLADDER UROTHELIAL CARCINOMA}

Nafissa El Badadawy, Nermeen Youssef, Amira Ismail, Faten Ragheb, Sara Hakim

Department of Pathology, Faculty of Medicine, Ain Shams

University, Egypt
Background: EGFR (ERB B1) and HER4 (ERB B4) are members of the ERB $B$ family of receptors. Overexpression of ERB $B$ receptors has both prognostic and therapeutic significance in various malignancies including breast, colorectal, prostate and ovarian cancer. However, the prognostic significance of their expression in urothelial carcinoma remains controversial. The aim of this work is to evaluate the prognostic value of EGFR and HER4 in urothelial carcinoma of urinary bladder.

Methods: Fifty archived cases of urothelial bladder carcinoma, cases of which had synchronous metastatic lesions in lymph nodes, as well as 13 samples of normal ureters were studied by immunostaining for EGFR and HER4.

Results: Membranous EGFR, cytoplasmic HER4 and nuclear HER4 expression were detected in $74 \%, 46 \%$ and $32 \%$ of urothelial carcinoma respectively and $15.4 \%, 100 \%$ and $0 \%$ of normal ureteric samples, repectively, with statistically significant differences between the two groups regarding the expression of both markers $(p<0.05)$. Membranous EGFR as well as nuclear HER4 expression showed statistically significant association with non-papillary tumors, high histologic grade and advanced tumor stage $(p<0.05)$. There was a highly statistically significant inverse relationship between cytoplasmic and nuclear HER4 expression $(p=0.0001)$ as well as between EGFR and cytoplasmic HER4 expression $(p=0.001)$. There was no statistically significant association between EGFR and nuclear HER4 expression $(p=0.051)$.

Conclusion: EGFR and nuclear HER4 expressions were associated with parameters of poor prognosi in urothelial carcinoma while cytoplasmic HER4 expression was related to good prognostic indicators. Both markers may help in selecting high-risk patients for more aggressive therapy.

\section{HISTOPATHOLOGIC AND SIASCOPIC CORRELATION OF PIGMENTED SKIN LESIONS IN RURAL NSW: A TWO YEAR PROSPECTIVE STUDY}

$\underline{\text { Sujatha Fernando }}{ }^{1}$, Fong Seen $\mathrm{Koh}^{1}$, Anna Durie ${ }^{1}$, Anne Gilroy ${ }^{2}$

${ }^{1}$ Southern IML, and ${ }^{2}$ University of Western Sydney, Australia

A prospective study was undertaken in collaboration with primary care physicians from skin clinics in rural New South Wales, which included Orange, Bathurst, Kelso and Illawong. Pigmented skin lesions, thought to be suspicious of atypical naevomelanocytic lesions, were subjected to spectrophotometric intracutaneous analysis (SIA) prior to excisional biopsy and histopathologic examination.

The simple, highly reproducible features, such as dermal melanin, collagen holes and 'erythematous blush' with blood displacement, found to be specific for melanoma by a previous study by Moncrief et al. ${ }^{1}$ in the United Kingdom was used by us. Analysis of our study over a period of two years is compared with the previous study by Moncrieff et al. ${ }^{1}$

Our results show that of 1014 pigmented skin lesions examined, 756 cases were identified as naevomelanocytic, with 449 dysplastic and 10 cases of melanoma. Of the non-melanocytic lesions, the most common, clinically misdiagnosed for atypical naevomelanocytic lesions, were seborrheic keratoses, similar to the UK study. 